\title{
Metabolic Syndrome Prevalence and Risk in the United States based on NHANES 2001-2012 Data
}

\author{
Brian Miller ${ }^{1,2^{*}}$ and Mark Fridline ${ }^{3}$ \\ ${ }^{1}$ School of Sport Science and Wellness Education, The University of Akron, Akron, OH, USA \\ ${ }^{2}$ Health Education and Promotion, School of Health Sciences, Kent State University, Kent, OH, USA \\ ${ }^{3}$ Department of Statistics, The University of Akron, Akron, OH, USA
}

"Corresponding author: Brian Miller, The University of Akron, School of Sport Science and Wellness Education, InfoCision Stadium 317, Akron OH, USA, Tel: 216-659-6985; E-mail: bm25@zips.uakron.edu

Rec date: April 26, 2016; Acc date: May 19, 2016; Pub date: May 26, 2016

Copyright: (c) 2016 Miller B, et al. This is an open-access article distributed under the terms of the Creative Commons Attribution License, which permits unrestricted use, distribution, and reproduction in any medium, provided the original author and source are credited.

\begin{abstract}
Purpose: The purpose of the current investigation was to assess Metabolic Syndrome prevalence and risk estimates using United States nationally representative data.

Methods: Study sample was derived from 6 National Health and Nutrition Examination Survey (NHANES) cohorts from 2001-2012, $N=9,326$ (male: $n=4,814$; female: $n=4,512$ ) including ages 18-59 presenting as fasted for 12 hours prior to laboratories collection. Variables included AHA/NHBLI Metabolic Syndrome classification criteria as well as additional cardiometabolic measures. Prevalence of Metabolic Syndrome and risk factors across cohorts as well as relative risk estimates were derived. Estimates were adjusted for age, race, and sex.

Results: There was no statistically significant difference between Metabolic Syndrome prevalence across cohorts. The order of Metabolic Syndrome criteria from highest to lowest risk were waist circumference, triglycerides, $\mathrm{HDL}$, fasting plasma glucose, and blood pressure for the total sample and across sex, with women presenting with larger risk estimates than men. Women had larger prevalence of waist circumference, HDL, and blood pressure risk factors compared to men who had a larger prevalence of triglyceride and fasting plasma glucose risk factors. Those presenting with Metabolic Syndrome were twice as likely to have a cardiovascular event.
\end{abstract}

Conclusion: Waist circumference and triglycerides were the Metabolic Syndrome risk factors with the highest prevalence and associated risk of developing Metabolic Syndrome. Those with Metabolic Syndrome were at increased risk of having a cardiovascular event.

Keywords: Cardiovascular disease; Metabolic syndrome; Waist circumference; NHANES; Risk

\section{Introduction}

Metabolic Syndrome (MetS) is a constellation of cardio-metabolic risk factors that, when present in tandem, increase cardiovascular morbidity and/or mortality [1-3]. The high prevalence of cardiometabolic risk factors in MetS threatens to undermine all recent gains to prevent and control related chronic disease [4]. Originally described in 1988 by Gerald Reaven as Syndrome X, MetS was classified by the interrelationship between inflammation, impaired fibrinolysis, hypertension, atherogenic dyslipidemia, visceral obesity, and dysglycemia and their association with developing chronic diseases including cardiovascular disease (CVD), insulin resistance (IR), and hypertension (HTN) [5]. The prevalence and complications associated with MetS have remained a major health concern in the US $[5,6]$.

The classification of MetS is based on the presence of 3 of 5 risk factors including dyslipidemia characterized by increased triglycerides (TG) and decreased HDL-cholesterol (HDL-C), hypertension, hyperglycemia, and central obesity. Current classification models [National Health Lung and Blood Institute (AHA/NHLBI), National
Cholesterol Education Program (NCEP), and International Diabetes Federation (IDF)] have been limited in their usefulness given that each is only able to identify the presence or absence of MetS rather than identifying changes in risk of developing MetS [7-11] and related cardiovascular mortality [12]. Presently, the National Health Lung and Blood Institute (AHA/NHLBI) classification criteria are the accepted classification protocol for MetS. (Table 1) [1].

Given the limitations of the MetS classification and the dynamic nature of cardio-metabolic disease research, current nationally representative risk estimates based on the current MetS classification criteria and additional cardiometabolic risk factors are necessary to inform future research and clinical practice. To date, there have been numerous reports on the prevalence and associated risk of MetS $[2,12,13]$. However these systematic reviews present risk and prevalence statistics using antiquated data. Numerous studies have presented MetS prevalence statistics derived from multiple classification criteria using data from the National Health and Nutrition Examination Survey (NHANES), (Table 1). However, these studies were based on limited amounts of data utilizing few representative cohorts with Beltrán-Sánchez et al. utilizing the most NHANES cohorts [14-18]. 


\begin{tabular}{|l|l|l|l|}
\hline Study & Cohort Years & $\begin{array}{l}\text { Classificat } \\
\text { ion } \\
\text { Criteria }\end{array}$ & $\begin{array}{l}\text { Mets } \\
\text { Prevalence }\end{array}$ \\
\hline $\begin{array}{l}\text { Mozumdar and Liguori } \\
{[15]}\end{array}$ & NHANES III & NCEP & $\begin{array}{l}27.9 \% \quad \text { NHANES } \\
\text { III }\end{array}$ \\
\hline & NHANES 1999-2006 & $\begin{array}{l}34.1 \% \quad \text { NHANES } \\
1999-2006\end{array}$ \\
\hline $\begin{array}{l}\text { Beltrán-Sanchez et al. } \\
{[14]^{*}}\end{array}$ & NHANES 1999-2010 & IDF & $25.5-22.9 \%$ \\
\hline Ford et al. [16]* & NHANES III & NCEP & $\begin{array}{l}23.1 \% \quad \text { NHANES } \\
\text { III }\end{array}$ \\
\hline & NHANES 1999-2000 & & $\begin{array}{l}26.7 \% \quad \text { NHANES } \\
1999-2000\end{array}$ \\
\hline Ford et al. [3] & NHANES 2003-2006 & NCEP & $34.10 \%$ \\
\hline Miller and Fridline [17] & NHANES 2009-2010 & NCEP & $33.10 \%$ \\
\hline *Age-Adjusted & \multicolumn{2}{l}{} \\
\hline
\end{tabular}

Table 1: Select MetS Publications Reporting Prevalence using NHANES data.

The purpose of the current investigation was to develop MetS prevalence and risk estimates using a nationally representative data from NHANES 2001-2012 cohorts. This study was guided by 2 aims. The first aim was to identify the prevalence of MetS in the United States general population using NHANES data from 2001-2012. The second aim was to identify MetS risk based on AHA/NHLBI classification criteria, sex, and cardiovascular events.

\section{Methods}

\section{Data management}

The study sample was derived from National Health and Nutrition Examination Survey (NHANES) data made publically available by the Centers for Disease Control and Prevention which included cohorts. Data was collected from 2001-2012 in 2 year intervals resulting in a total of 6 cohorts. The data was arranged in a column-wise format with each subject given a sequence identifier. Data management was performed using dataset merging and data subset functions using SPSS version 22 (SPSS Inc., Chicago, IL).

The inclusion criteria were based on the following parameters: Age range of 18-59 years, 12 hour fasting protocol for laboratory values, abstinence from alcohol and/or tobacco use prior to laboratories, and a negative exam for pregnancy for females. The age criteria was chosen based on Ford Li, and Zhao where the highest prevalence of MetS was exhibited after 59 years of age [3]. Inclusion of ages beyond 59 resulted in inflation of risk estimates. The MetS classification was defined as the presence of 3 of 5 risk factors based on the clinical classification model proposed by the AHA/NHLBI (Table 2) [1] Subjects with missing criteria were excluded from the analysis unless the criteria present were adequate to make a MetS classification. This decision was made in order to control for the inability to make a complete classification. Blood pressure readings were the average of 4 blood pressure collections per subject. The presence of each MetS classification criteria was dichotomized with $\geq 3$ of 5 criteria classified as MetS. The final sample size for inclusion was $\mathrm{N}=9,326$ (male: $\mathrm{n}=4,814$; female: $\mathrm{n}=$
4,512). The current investigation was approved by the Institutional Review Board of the University of Akron.

\begin{tabular}{|c|c|}
\hline Measure & Defining Cut-off Points \\
\hline \multicolumn{2}{|c|}{ Elevated Waist Circumference ${ }^{1}$} \\
\hline Male & $>94 \mathrm{~cm}$ \\
\hline Female & $>80 \mathrm{~cm}$ \\
\hline Elevated Triglycerides ${ }^{2}$ & $\geq 150 \mathrm{mg} / \mathrm{dl}$ \\
\hline \multicolumn{2}{|l|}{ HDL Cholesterol $^{2}$} \\
\hline Male & $<40 \mathrm{mg} / \mathrm{dl}$ \\
\hline Female & $<50 \mathrm{mg} / \mathrm{dl}$ \\
\hline Blood Pressure ${ }^{2}$ & $\geq 130 \mathrm{mmHg}$ Systolic and/or $\geq 80 \mathrm{mmHg}$ Diastolic \\
\hline Fasting Plasma Glucose ${ }^{2}$ & $\geq 100 \mathrm{mg} / \mathrm{dl}$ \\
\hline \multicolumn{2}{|c|}{${ }^{1}$ Values based on lowered AHA/NHLBI Guidelines (Alberit) } \\
\hline \multicolumn{2}{|c|}{$\begin{array}{l}{ }^{2} \text { Drug therapy for dyslipidemia, hypertension, and/or hyperglycemia wer } \\
\text { alternate indicators meeting the criteria for MetS for that risk factor }\end{array}$} \\
\hline
\end{tabular}

Table 2: National Health Lung \& Blood Institute Metabolic Syndrome Classification Criteria.

In addition to the AHA/NHLBI MetS classification criteria, indicators of cardio-metabolic morbidity included: a binary indicator of cardiovascular events built off of the presence of 1 of 5 cardiovascular events including congestive heart failure, coronary heart disease, angina, heart attack, and/or stroke [19]. Descriptive statistics included the above mentioned measure were presented as $\mathrm{M}$ \pm SD in addition to the following measures: HOMA-IR, an indicator of insulin resistance defined as [Insulin ( $\mathrm{uU} / \mathrm{dl})$ x $\mathrm{FPG}(\mathrm{mg} / \mathrm{dl}) / 405$ ] $[6,18,19]$, cardio-metabolic risk factors [total cholesterol (TC), LDL cholesterol, and C-Reactive Protein (CRP) - systemic inflammatory marker [20], anthropometrics [height $(\mathrm{cm})$, weight $(\mathrm{kg})$, and Body Mass Index (BMI) $\left(\mathrm{kg} / \mathrm{m}^{2}\right)[8,21]$; and socioeconomic status measured via Family Poverty to Income ratio (PIR) a measure of adjusted family income to relative poverty threshold based on house size [22].

\section{Sample adjustment}

Sample weights were created in NHANES to account for the complex sample design when capturing participant data. This sample design included survey non-response, post-stratification, and oversampling certain demographic groups. When a sample is weighted using NHANES data, the results are representative of the U.S. Census civilian non-institutionalized population. A sample weight is assigned to each person in the sample, where it measures the frequency of people in the population represented by that sample individual. It is important to utilize the weights to ensure that the calculated parameter estimates are truly representative of our population. To account for the complex survey design, statistical results were calculated according to NHANES guidelines [23]. The SPSS Complex Sampling module was used to take into account for the NHANES complex survey design.

\section{Statistical analysis}

A Chi-Squared $\left(\mathrm{X}^{2}\right)$ test of goodness of fit was employed to indentify differences in the prevalence of MetS by NHANES cohort 
Citation: Miller B, Fridline M (2016) Metabolic Syndrome Prevalence and Risk in the United States based on NHANES 2001-2012 Data. J

Page 3 of 6

year. Additionally in cases where the $\mathrm{X}^{2}$ test reached statistical significance, post hoc tests were performed using the standardized residuals (SR) with $\mathrm{SR}>2$ indicating significant deviations. Risk estimates [relative risk (RR) accompanied with 95\% confidence intervals] were produced for each MetS classification criteria as well as
Cardiovascular Events and Sex in relation to MetS. All statistical analysis were performed using SPSS version 22 (IBM, Chicago, IL, 2013) with statistical significance for all tests set at $p \leq 0.05$ (Table 3 ).

\begin{tabular}{|c|c|c|c|c|c|c|c|}
\hline & & No MetS & & & MetS & & \\
\hline Variable & Sample & $\mathbf{n}$ & $\mathbf{M}$ & SD & $\mathbf{n}$ & $\mathbf{M}$ & SD \\
\hline \multirow[t]{2}{*}{ Age (years) } & Male & 3543 & 33.05 & 12.09 & 1931 & 42.48 & 11.11 \\
\hline & Female & 3570 & 34.41 & 11.87 & 1595 & 43.23 & 11.31 \\
\hline \multirow[t]{2}{*}{ Family PIR } & Male & 3278 & 2.55 & 1.63 & 1806 & 2.69 & 1.67 \\
\hline & Female & 3285 & 2.53 & 1.68 & 1460 & 2.35 & 1.64 \\
\hline \multirow[t]{2}{*}{ Height (cm) } & Male & 3542 & 175.57 & 7.77 & 1931 & 175.79 & 7.72 \\
\hline & Female & 3570 & 162.43 & 6.96 & 1592 & 161.71 & 7.02 \\
\hline \multirow[t]{2}{*}{ BMI } & Male & 3542 & 25.83 & 4.83 & 1931 & 31.37 & 5.65 \\
\hline & Female & 3570 & 26.54 & 6.25 & 1592 & 33.13 & 7.57 \\
\hline \multirow[t]{2}{*}{$\mathrm{CRP}(\mathrm{mg} / \mathrm{dL})$} & Male & 3003 & 0.25 & 0.72 & 1645 & 0.41 & 0.77 \\
\hline & Female & 3027 & 0.36 & $68.00 \%$ & 1357 & 0.72 & 0.97 \\
\hline \multirow[t]{2}{*}{$\mathrm{TC}(\mathrm{mg} / \mathrm{dL})$} & Male & 3543 & 186.22 & $3928.00 \%$ & 1931 & 205.24 & 45 \\
\hline & Female & 3570 & 186.46 & 37.6 & 1595 & 204.27 & 45.07 \\
\hline \multirow[t]{2}{*}{ LDL-C (mg/dL) } & Male & 3462 & 113.67 & 34.55 & 1729 & 123.55 & 36.22 \\
\hline & Female & 3512 & 108.52 & 31.87 & 1512 & 122.15 & 36.89 \\
\hline \multirow[t]{2}{*}{ WC $(\mathrm{cm})$} & Male & 3543 & 90.78 & 13.32 & 1931 & 107.89 & 13.85 \\
\hline & Female & 3570 & 88.24 & 14.3 & 1595 & 104.93 & 15.42 \\
\hline \multirow[t]{2}{*}{$\mathrm{TG}(\mathrm{mg} / \mathrm{dL})$} & Male & 3543 & 105.17 & 68.16 & 1931 & 224.58 & 212.46 \\
\hline & Female & 3570 & 87.23 & 40.54 & 1595 & 178.77 & 144.36 \\
\hline \multirow[t]{2}{*}{$\mathrm{HDL}-\mathrm{C}(\mathrm{mg} / \mathrm{dL})$} & Male & 3543 & 51.7 & 13.37 & 1931 & 40.86 & 10.59 \\
\hline & Female & 3570 & 60.39 & 14.59 & 1595 & 47.74 & 13.36 \\
\hline \multirow[t]{2}{*}{$\mathrm{SBP}(\mathrm{mmHg})$} & Male & 3543 & 117.68 & 11.59 & 1931 & 126.54 & 15.03 \\
\hline & Female & 3570 & 110.92 & 12.26 & 1595 & 123.38 & 17.7 \\
\hline \multirow[t]{2}{*}{$\mathrm{DBP}(\mathrm{mmHg})$} & Male & 3543 & 69.35 & 11.08 & 1931 & 77.04 & 11.67 \\
\hline & Female & 3570 & 67.34 & 9.55 & 1595 & 73.79 & 11.24 \\
\hline \multirow[t]{2}{*}{ FPG (mg/dL) } & Male & 3543 & 96.51 & 18.47 & 1931 & 118.46 & 47.53 \\
\hline & Female & 3570 & 91.73 & 14.3 & 1595 & 114.9 & 43.92 \\
\hline \multirow[t]{2}{*}{ HOMA-IR } & Male & 3500 & 2.37 & 2.08 & 1917 & 5.96 & 8.49 \\
\hline & Female & 3530 & 2.29 & 1.72 & 1577 & 5.45 & 5.48 \\
\hline
\end{tabular}

Table 3: Descriptive Statistics for Total NHANES Cohort and by Sex. 
Citation: Miller B, Fridline M (2016) Metabolic Syndrome Prevalence and Risk in the United States based on NHANES 2001-2012 Data. J

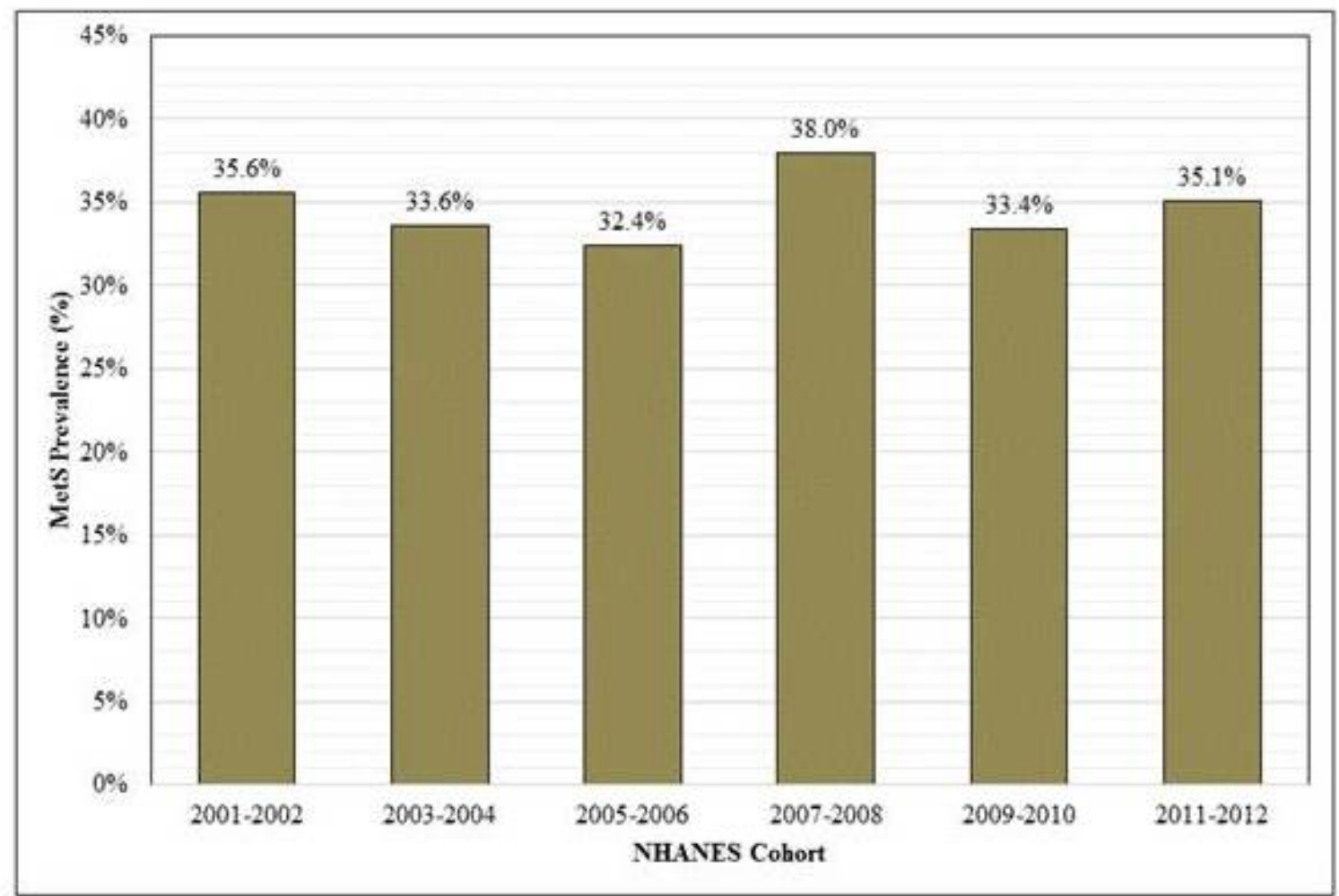

Figure 1: Prevalence of MetS classified by AHA/NHLBI criteria for each NHANES cohort year.

\section{Results}

\section{Prevalence of MetS}

Table 3 depicts the sample characteristics by MetS and no-MetS. There was not a statistically significant difference in prevalence of MetS by NHANES cohort year, $\chi^{2}(6)=13.83, \mathrm{p}=0.257$.

The average prevalence of MetS in the total cohort was $34.7 \pm 1.4 \%$. Figure 1 for MetS prevalence by NHANES cohort year.

Based on the indicators of MetS risk based on the presence of each risk factor (see Table 4 for total cohort and by sex), the order of highest to lowest risk were WC, TG, HDL, FPG, and BP for the total sample and across sex, respectively. However, females had larger risks than men for all MetS risk factors. The risk factor with the largest difference by sex was WC with women being on average 14.11 times more likely to develop MetS compare to men; RR = 23.8 (14.58-38.77) compared to $\mathrm{RR}=9.69$ (7.93-11.86) for men and women respectively. Women had a larger prevalence of risk WC, HDL, and BP risk factors compared to men who had a larger prevalence of TG and FPG risk factors. Specifically the risk factor with the largest prevalence was WC representing $77 \%$ of women and $61 \%$ men. There was a 2 fold increase in risk of a cardiovascular event given the presence of metabolic syndrome for the total sample $[R R=2.02(1.83-2.23)]$ with a higher risk for men $[R R=1.81(1.54,2.13)]$ compared to women $[R R=2.32$ (2.01-2.68)].

\section{Discussion}

Metabolic Syndrome is a constellation of cardiometabolic risk factors that when present in tandem increase the risk morbidity and mortality $[1,3]$. The current investigation was guided by 2 aims. The first aim explored the prevalence of MetS by NHANES cohort year from 2001-2012. Differences in prevalence rates of the current investigation compared to other studies is likely based on differences in MetS classification criteria, subject inclusion criteria, and adjustments made for age. The MetS prevalence of this study (34.7\%) approximates the prevalence of MetS for Ford et al. $=34.1 \%$, Miller and Fridline $=$ $33.1 \%$, and Mozumdar and Liguori $=34.1 \%$. Beltrán-Sánchez et al. reported an average prevalance of MetS using NHANES data at 25.5\% in 1999 and $22.9 \%$ in 2010. However this article used an ageadjustment and did not restrict age.

The second aim was to identify MetS risk based on AHA/NHLBI classification criteria, sex, and history of cardiovascular events. The risk factor with the largest risk of MetS was WC with a larger impact for men than women. This results corroborates with Miller et al. who found WC as the strongest predictor of MetS using a decision tree algorithm. Additionally, Beltrán-Sánchez et al. reported an increase in abdominal obesity, with a more drastic increase among women. Increased WC has been used as a screening phenotype to identify those at high risk of MetS [24]. This study identified TG as the second strongest indicator of MetS risk with 32\%, 38\%, and 25\% for the total cohort, male, and female presenting with this risk factor, respectively.

Miller and Fridline and Worachartcheewan et al. both identified that the TG criteria resulted in the highest risk of MetS. However based 
Page 5 of 6

on risk estimates of the currefnt investigation, TG and HDL had less risk than WC. Dyslipidemia in MetS, defined as high serum TG and low HDL-C, have been demonstrated as effective markers for the presence of cardio-metabolic abnormalities [24]. An analysis of NHANES 2003-2006 data showed that $31 \%$ of adults exhibit hypertriglyceridemia (>150 mg/dl) [3]. Beltrán-Sánchez et al. reported an increase in dyslipidemia, hyperglycemia, and waist circumference from 1999-2010 with a decrease prevalence in hyper tension.

\begin{tabular}{|c|c|c|c|c|}
\hline Variable & Sample & Proportion & $\mathbf{R R}$ & $\begin{array}{l}95 \% \text { Confidence } \\
\text { Interval }\end{array}$ \\
\hline \multirow[t]{3}{*}{ WC } & Total & 0.68 & 9.69 & $7.93-11.86$ \\
\hline & Male & 0.61 & 8.68 & $6.97-10.82$ \\
\hline & Female & 0.77 & 23.8 & $14.58-38.77$ \\
\hline \multirow[t]{3}{*}{ TG } & Total & 0.32 & 5.69 & $5.29-6.12$ \\
\hline & Male & 0.38 & 5.14 & $4.62-5.70$ \\
\hline & Female & 0.25 & 6.38 & $5.71-7.13$ \\
\hline \multirow[t]{3}{*}{ HDL } & Total & 0.35 & 4.93 & $4.59-5.29$ \\
\hline & Male & 0.33 & 4.3 & $3.93-4.71$ \\
\hline & Female & 0.37 & 6.31 & $5.50-7.24$ \\
\hline \multirow[t]{3}{*}{ FPG } & Total & 0.37 & 4 & $3.69-4.34$ \\
\hline & Male & 0.45 & 3.34 & $2.99-3.72$ \\
\hline & Female & 0.28 & 4.88 & $4.35-5.49$ \\
\hline \multirow[t]{3}{*}{$\mathrm{BP}$} & Total & 0.28 & 3.58 & $3.36-3.81$ \\
\hline & Male & 0.32 & 3.15 & $2.87-3.45$ \\
\hline & Female & 0.24 & 4.1 & $3.78-4.46$ \\
\hline \multirow[t]{3}{*}{ CE } & Total & 0.04 & 2.02 & $1.83-2.25$ \\
\hline & Male & 0.04 & 1.81 & $1.54-2.13$ \\
\hline & Female & 0.03 & 2.32 & $2.01-2.68$ \\
\hline
\end{tabular}

Table 4: Adjusted MetS Risk Statistics for the Total Cohort and by Sex

Després et al. found that a tandem increase of TG above $2 \mathrm{mmol} / \mathrm{L}$ with a WC greater than $90 \mathrm{~cm}$ showed a greater than $80 \%$ increase in risk of developing MetS. Although there was not a large difference in MetS risk by sex, the order of risk contribution for each MetS criteria to MetS risk were different. This supports the need for future investigation to consider differences in cardio-metabolic risk by sex as well as the clustering interaction of MetS risk factors. Specific to cardiovascular risk in the current investigation was approximately 2 times greater for those with MetS. This finding corroborates with Mottillo et al. who found that MetS was associated with a 2 -fold increase risk of cardiovascular events in addition to a 1.5 fold increase in all-cause mortality.

The results of this study are meant to provide risk estimates for MetS across multiple cardiometabolic risk factors and differences by sex.
Future investigation should employ these estimates to guide future investigation for MetS risk factors investigated. The predominant finding of the current investigation was the drastic risk increase of MetS based on presenting with WC risk factor. Furthermore there was a large disparity for women compared to men based on presenting with the WC risk factor. The results of this article in tandem with recommendations from Beltrán-Sánchez et al., Miller and Fridline and Miller et al. emphasizes the urgency of recognizing abdominal obesity as a healthcare priority.

This study had numerous strengths which included the use of large amounts of nationally representative data and described contributions to MetS risk. Beltran-Sanchez et al. utilized data from the 1999-2010 NHANES cohorts to explore the prevalence of the MetS classification criteria across race, age, and sex. However this study did not investigate the contribution of cardio-metabolic risk factors to overall MetS risk. The current investigation study also had limitations. This study did not employ an age-adjustment to describe prevalence and risk of MetS but rather an adjustment across sex and race. This study also relied on secondary, observational data where differences in data collection and analysis were not accounted for.

In summary, the purpose of this study was to explore differences in MetS prevalence across 6 NHANES cohorts and MetS risk for MetS classification criteria and additional cardio-metabolic risk factors. There were differing levels MetS risk for each of the cardiometabolic risk factors for the total sample and by sex. The risk factor with the highest proportion and risk for the total sample and by sex was WC. Women had a higher prevalence of WC, HDL, and BP risk factors compared to men who had a higher prevalence of TG and FPG. Presenting with the WC risk factor had the largest associated risk with MetS with a larger risk for women compared to mean. Future research should utilize these findings to guide experimental research considering MetS risk [25,26].

\section{Conflict of Interest}

The author of this study declared no conflict of interest.

\section{References}

1. Alberti KGMM, Eckel RH, GrundySM, Zimmet PZ, CleemanJI, et al. (2009) Harmonizing the metabolic syndrome: A joint interim statement of the international diabetes federation task force on epidemiology and prevention; national heart, lung, and blood institute; american heart association; world heart federation; international atherosclerosis society; and international association for the study of obesity. Circulation 120: 1640-1645.

2. Eberly LE, Prineas R, Cohen JD, Vazquez G, Zhi X, et al. (2006) Metabolic syndrome risk factor distribution and 18-year mortality in the Multiple Risk Factor Intervention Trial. Diabetes Care 29: 123-130.

3. Ford ES, Li C, Zhao G (2010) Prevalence and correlates of metabolic syndrome based on a harmonious definition among adults in the US. J Diabetes 2: 180-193.

4. Eckel RH, Grundy SM, Zimmet PZ (2005) The metabolic syndrome. The Lancet 365: 1415-1428.

5. Grundy SM (2011) The metabolic syndrome. Atlas of atherosclerosis and metabolic syndrome (5th edn), NY: Springer, New York.

6. Wilson PW, D'Agostino RB, Parise H, Sullivan L, Meigs JB (2005) Metabolic syndrome as a precursor of cardiovascular disease and type 2 diabetes mellitus. Circulation 112: 3066-3072.

7. Alberti KG, Zimmet P, Shaw J; IDF Epidemiology Task Force Consensus Group (2005) The metabolic syndrome-a new worldwide definition. Lancet 366: 1059-1062. 
Citation: Miller B, Fridline M (2016) Metabolic Syndrome Prevalence and Risk in the United States based on NHANES 2001-2012 Data. J

8. Arnlov J, Ingelsson E, Sundström J, Lind L (2010) Response to letter regarding article,"The impact of body mass index and the metabolic syndrome on the risk of cardiovascular disease and death in middle-aged men". Circulation 122: e457-e457.

9. Boudreau DM, Malone DC, Raebel MA, Fishman PA, Nichols GA, et al. (2009) Health care utilization and costs by metabolic syndrome risk factors. Metab Syndr Relat Disord 7: 305-314.

10. Kassi E, Pervanidou P, Kaltsas G, Chrousos G (2011) Metabolic syndrome: definitions and controversies. BMC Med 9: 48.

11. Miller B, Fridline M, Liu PY, Marino D (2014) Use of CHAID decision trees to formulate pathways for the early detection of metabolic syndrome in young adults. Computational and mathematical methods in medicine.

12. Mottillo S, Filion KB, Genest J, Joseph L, Pilote L, et al. (2010) The metabolic syndrome and cardiovascular risk a systematic review and meta-analysis. J Am Coll Cardiol 56: 1113-1132.

13. Gami AS, Witt BJ, Howard DE, Erwin PJ, Gami LA, et al. (2007). Metabolic syndrome and risk of incident cardiovascular events and death a systematic review and meta-analysis of longitudinal studies. Journal of the American College of Cardiology 49: 403-414.

14. Beltrán-Sánchez H, Harhay MO, Harhay MM, McElligott S (2013) Prevalence and trends of metabolic syndrome in the adult U.S. population, 1999-2010. J Am Coll Cardiol 62: 697-703.

15. Mozumdar A, Liguori G (2011) Persistent increase of prevalence of metabolic syndrome among U.S. adults: NHANES III to NHANES 1999-2006. Diabetes Care 34: 216-219.

16. Ford ES, Giles WH, Mokdad AH (2004) Increasing prevalence of the metabolic syndrome among u.s. Adults. Diabetes Care 27: 2444-2449.

17. Miller B, Fridline M (2015) Development and Validation of Metabolic Syndrome Prediction and Classification-Pathways using Decision Trees. J Metabol Synd 4: 2167-0943.

18. Angelico F, Burattin M, Alessandri C, Del Ben M, Lirussi F (2007) Drugs improving insulin resistance for non-alcoholic fatty liver disease and/or non-alcoholic steatohepatitis. The Cochrane Library.
19. Ninomiya JK, L'Italien G, Criqui MH, Whyte JL, Gamst A, et al. (2004). Association of the metabolic syndrome with history of myocardial infarction and stroke in the third national health and nutrition examination survey. Circulation 109: 42-46.

20. Nayak BS, Teelucksingh S, Jagessar A, Maharaj S, Maharaj N (2013) A cross sectional study comparing traditional risk factors with $\mathrm{N}$-terminal pro-BNP in high risk groups for cardiovascular disease in Trinidad, West Indies. Diabetes \& Metabolic Syndrome: Clinical Research \& Reviews 7: 8-11.

21. Camhi SM, Bray GA, Bouchard C, Greenway FL, Johnson WD, et al. (2011) The relationship of waist circumference and BMI to visceral, subcutaneous, and total body fat: sex and race differences. Obesity (Silver Spring) 19: 402-408.

22. Matthews KA, Räikkönen K, Gallo L, Kuller LH (2008) Association between socioeconomic status and metabolic syndrome in women: testing the reserve capacity model. Health Psychology 27: 576.

23. National Center for Health Statistics (2008) Specifying Weighting Parameters. Atlanta, GA:Centers for Disease Control and Prevention, NCHS.

24. Roger VL, Go AS, Lloyd-Jones DM, Adams RJ, Berry JD, et al. (2011) Heart disease and stroke statistics-2011 update a report from the American Heart Association. Circulation 123: e18-e209.

25. Worachartcheewan A, Dansethakul P, Nantasenamat C, Pidetcha P, Prachayasittikul V (2012) Determining the optimal cutoff points for waist circumference and body mass index for identification of metabolic abnormalities and metabolic syndrome in urban Thai population. Diabetes Research and Clinical Practice 98: e16-e21.

26. Despres JP, Lemieux I, Bergeron J, Pibarot P, Mathieu P, et al. (2008) Abdominal obesity and the metabolic syndrome: Contribution to global cardiometabolic risk. Arterioscler Thromb Vasc Biol 28: 1039-1049. 